\title{
Surviving Case of a Blowout-Type Left Ventricular Free Wall Rupture During Percutaneous Coronary Intervention for a Lateral Acute Myocardial Infarction
}

\author{
Riku Arai, ${ }^{1}$ MD, Daisuke Fukamachi, ${ }^{1}$ MD, Naotaka Akutsu, ${ }^{1}$ MD, Masashi Tanaka, ${ }^{2}$ MD and \\ Yasuo Okumura, ${ }^{1}$ MD
}

\begin{abstract}
Summary
A 76-year-old man suffering from chest pain was admitted to our hospital with a suspected acute myocardial infarction (AMI). Emergent coronary angiography revealed a totally occluded proximal left circumflex artery (LCX). During primary percutaneous coronary intervention, his blood pressure suddenly fell within seconds, and he developed pulseless electrical activity (PEA). Surprisingly, the 12-lead electrocardiogram (ECG) findings including the heart rate remained unchanged before and after the PEA, but a heart rate reduction and asystole occurred a few minutes after developing PEA. After tracheal intubation and mechanical assistance by venoarterial extracorporeal membrane oxygenation (VA-ECMO), the sudden onset of PEA appeared to be caused by cardiac tamponade due to a blowout-type left ventricular free wall rupture (BO-LVFWR) diagnosed by transthoracic echocardiography. While pericardiocentesis was performed and the drained blood was directly continuously perfused intravenously to keep the VA-ECMO flow, the patient was moved to the operation room. The surgical findings revealed a solitary BO-LVFWR due to a lateral AMI, and a direct closure was performed. Successful perioperative management, oral medication administration, and rehabilitation lead to the patient being transferred to a rehabilitation hospital without any serious cerebral damage. This case report suggested the detailed onset pattern of a BO-LVFWR followed by a rapid diagnosis by echocardiography and lifesaving treatment.
\end{abstract}

(Int Heart J 2020; 61: 606-610)

Key words: Transthoracic echocardiography, Mechanical circulatory support, Continuous vital sign monitoring

A blowout-type left ventricular free wall rupture (BO-LVFWR) is a fatal complication of an acute myocardial infarction (AMI). ${ }^{1)}$ Treatment of this complication remains challenging due to the limited time to perform further lifesaving therapeutic interventions following the onset of the BO-LVFWR. We herein experienced a case of a BO-LVFWR occurring during percutaneous coronary intervention (PCI) for an AMI, which could be diagnosed and treated early and eventually discharged without any serious cerebral damage.

\section{Case Report}

A 76-year-old man with hypertension experienced a sudden onset of chest pain. The chest pain persisted, and he was transferred to our hospital 6 hours after the onset. There were no signs of shock according to his vital signs and physical findings during the initial examination (Table I). A synthesized 18-lead electrocardiogram (ECG) during the initial examination revealed complete right bundle branch block with an elevated ST segment in leads I and
aVL and ST depression in leads III and aVF. Transthoracic echocardiography revealed a preserved global left ventricular function with slight hypokinesis due to a posterolateral lesion without a D-shape, echo-free space, or valvular disease. A chest X-ray revealed no notable findings, including congestion. The laboratory data showed increased creatine kinase (CK) and troponin I serum levels, which were checked during catheter examination. An AMI was diagnosed, and an emergent coronary angiography via the left radial artery revealed no significant stenosis in the right coronary artery, but a total occlusion in the proximal left circumflex artery (LCX), which coincided with the culprit inferred from the 18-lead ECG and echocardiography (Figure 1). We immediately moved to a primary PCI of the proximal LCX. First, we tried to wire the obstructed lesion in the LCX with a floppy wire (SION blue guide wire, Asahi Intecc Co., Ltd., Aichi, Japan); however, it did not pass due to the strong tortuosity and insufficient backup and penetration. Subsequently, wiring was attempted with a taper wire (XT-A guide wire, Asahi Intecc) under the support of a micro-catheter (Caravel MC

From the ${ }^{1}$ Division of Cardiology, Department of Medicine, Nihon University School of Medicine, Tokyo, Japan and ${ }^{2}$ Department of Cardiovascular Surgery, Nihon University School of Medicine, Tokyo, Japan.

Address for correspondence: Riku Arai, MD, Division of Cardiology, Department of Medicine, Nihon University School of Medicine, Oyaguchijomachi 30-

1, Itabashi-ku, Tokyo 173-8610, Japan. E-mail: riku.arai@icloud.com

Received for publication September 21, 2019. Revised and accepted December 25, 2019

Released in advance online on J-STAGE May 15, 2020.

doi: 10.1536/ihj.19-495

All rights reserved by the International Heart Journal Association. 
Table I. Vital Signs and the Lactate Level

\begin{tabular}{lcc}
\hline & At transport & At the time of the PCI \\
\hline Blood pressure (mmHg) & $179 / 101$ & $172 / 88$ \\
Heart rate $(\mathrm{bpm})$ & 60 & 95 \\
Respiratory rate (breaths/minute) & 17 & 16 \\
Body temperature (degree Celsius) & 37.3 & - \\
Consciousness level & clear & clear \\
Lactate (mmol/L) & 2.3 & - \\
\hline
\end{tabular}

PCI indidates percutaneous coronary intervention.

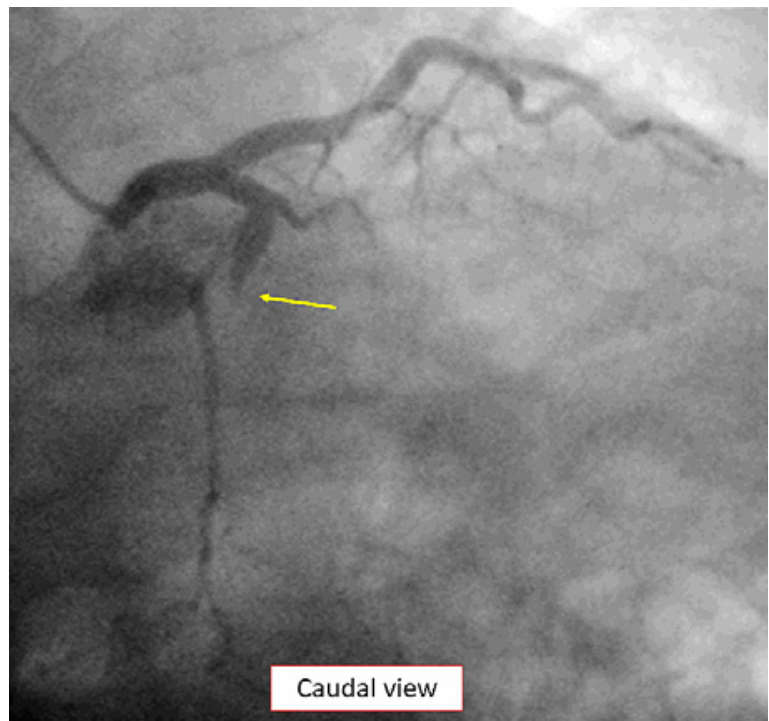

Figure 1. Coronary angiogram showing a total occlusion of the proximal left circumflex coronary artery (arrow).

micro-catheter, Asahi Intecc). However, his blood pressure suddenly fell within seconds while attempting to wire the obstructed lesion (Figure 2). When no cardiac output was present, cardiopulmonary resuscitation was started with a rapid response system. Rapid tracheal intubation by an emergency physician was performed, and venoarterial extracorporeal membrane oxygenation (VA-ECMO) via a right femoral approach was simultaneously established. The time from the cardiopulmonary arrest (CPA) to the VA-ECMO drive was 17 minutes (Table II). Surprisingly, the 12-lead ECG findings including the heart rate before and after the PEA remained unchanged (Figure 3), but a heart rate reduction and asystole occurred a few minutes after developing PEA. A temporary pacing catheter was immediately placed in the right ventricular apex via a left femoral approach, and right ventricular pacing was performed at $80 \mathrm{bpm}$. After establishing respiratory support by tracheal intubation and mechanical assistance by VAECMO and right ventricular pacing, the cause of the sudden onset of PEA was evaluated. Until the transthoracic echocardiography was ready, a left coronary angiogram was performed to rule out any vascular perforation due to wiring, but no extravasation was observed. Intravascular ultrasonography (IVUS) (Opticross, Boston Scientific, Boston Scientific Corp.) imaging from the left anterior descending coronary artery distal to the left main trunk ostium was performed to rule out a coronary artery dissection induced by the PCI procedure, but no significant dissection was found. On echocardiography, it was judged that the PEA was caused by cardiac tamponade because there was a pericardial effusion that was not recognized in the initial examination; thus, a pericardiocentesis was performed from an apex approach. The pericardial effusion was bloody (hemoglobin level was $12.0 \mathrm{~g} / \mathrm{dL}$ in a blood gas analysis in later), and the systemic blood pressure increased temporarily after drainage, but as soon as the drainage was stopped, the pericardial effusion reappeared, and the blood pressure decreased. When the amount of drainage exceeded $300 \mathrm{cc}$, it was judged that the cardiac tamponade was not caused by a coronary artery injury due to the PCI procedure but was due to a BO-LVFWR that complicated after the AMI, and we consulted a cardiovascular surgeon. An intra-aortic balloon pump (IABP) was placed via a left femoral approach, and a central venous catheter was placed from the right internal jugular vein. Up until the operation was ready, because blood transfusions were not rapidly available, in addition to the rapid infusion of extracellular fluid, a VA-ECMO flow was maintained by returning the drained blood directly from the central venous catheter placed in the right internal jugular vein. Eventually, the patient was able to be moved to the operation room while maintaining the VA-ECMO flow.

The surgical findings revealed a solitary BO-LVFWR due to a lateral AMI (Figure 4). There was a 2-cm rupture hole in the peripheral myocardium of the LCX. A direct closure was performed at the same site, and a central ECMO was attached to complete the procedure. The operation time was 244 minutes (Table II). After that, the CK level peaked out at $2895 \mathrm{IU} / \mathrm{L}$, and the myocardium stunned after the CPA and gradually improved day by day, and it was possible to withdraw him from the VAECMO 6 days after the surgery and the IABP 7 days after the surgery. Regarding the neurological course, convulsions were observed on the day after the operation, and a consultation was given by a neurologist. Based on the neurological examination, head computed tomography scan, and electroencephalogram findings, the patient was diagnosed with convulsions due to hypoxic encephalopathy and was treated with antiepileptic drugs. Considering the need for long-term artificial respiration management for the prolongation of a consciousness disorder due to convulsions, a tracheostomy was performed on the 19th postoperative day. After that, the convulsions disappeared, and the disturbance of consciousness improved until communication was achieved. Eventually, oral administration, 


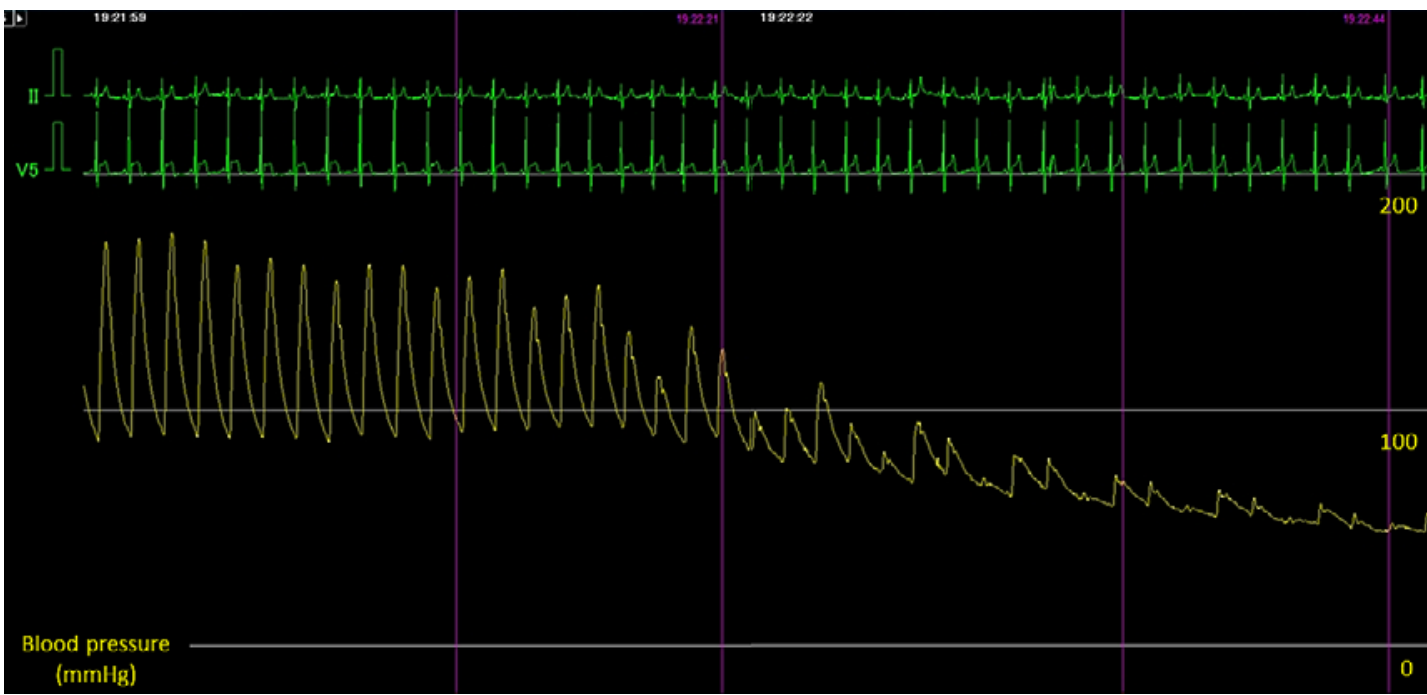

Figure 2. A sudden drop in blood pressure during percutaneous coronary intervention.

The blood pressure suddenly drops within seconds. Cardiopulmonary resuscitation is started 32 seconds after pulseless electrical activity.

Table II. Time Elapsed

\begin{tabular}{lc}
\hline & Elapsed time between \\
\hline Onset of chest pain & \\
$\downarrow$ & About 6 hours \\
Transport to our hospital & \\
$\downarrow$ & 35 minutes \\
Entering the catheter room & \\
$\downarrow$ & 34 minutes \\
Sudden drop in the blood pressure & \\
$\downarrow$ & 5 seconds \\
Developing PEA without any 12-lead ECG changes & \\
$\downarrow$ & 32 seconds \\
Starting cardiopulmonary resuscitation & \\
$\downarrow$ & \\
Developing bradycardia and asystole & About 5 minutes \\
$\downarrow$ & 17 minutes \\
Driving VA-ECMO & \\
$\downarrow$ & 16 minutes \\
Diagnosed with a BO-LVFWR & \\
$\downarrow$ & 199 minutes \\
Entering the operation room & \\
$\downarrow$ & 244 minutes \\
End of surgery & \\
\hline PEA indicates pulseless electrical activity; ECG, electrocardiogram; VA-ECMO, \\
venoarterial extracorporeal membrane oxygenation; and & BO-LVFWR, blow- \\
out-type left ventricular free wall rupture. &
\end{tabular}

including a beta-blocker and angiotensin II receptor blocker (ARB), and rehabilitation could be performed, and the patient was then transferred to a rehabilitation hospital on the 35th postoperative day for the purpose of continued rehabilitation.

\section{Discussion}

LVFWRs are clinically classified as either an oozing type or a blowout type. ${ }^{2)}$ The oozing type is characterized by a perforation, allowing enough time for a diagnosis and surgery, while the blowout type is characterized by rapid, irreversible, electromechanical dissociation, shock, and death within a few minutes due to massive hemorrhages into the pericardial cavity. According to the previous reports regarding LVFWRs, most cases are the oozing type, and blowout-type cases are relatively rare. ${ }^{2)}$ Although the blowout type is a rare but fatal complication of an AMI leading to sudden and devastating death, the clinical data for its characteristics and course are lacking. More- 
Before PEA: The time was 19:22:18.

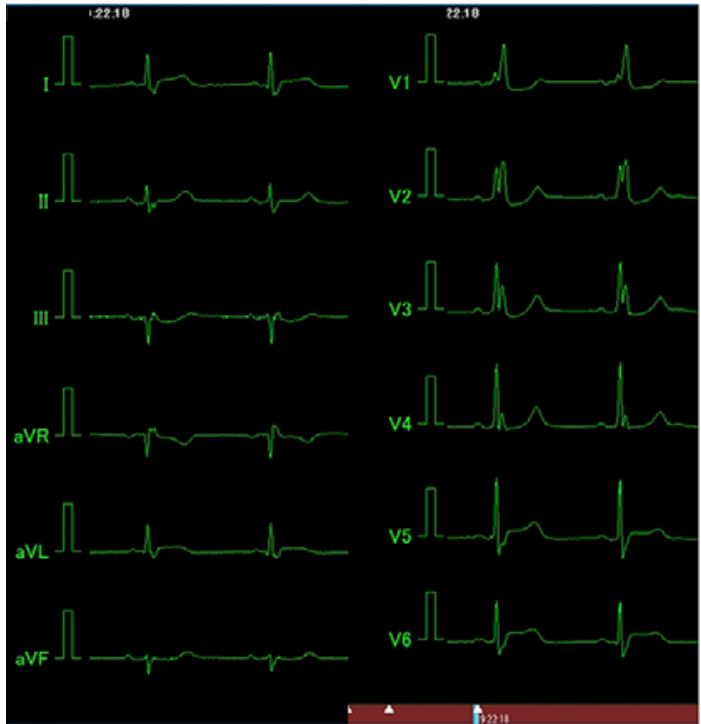

After PEA: The time was 19:22:56.

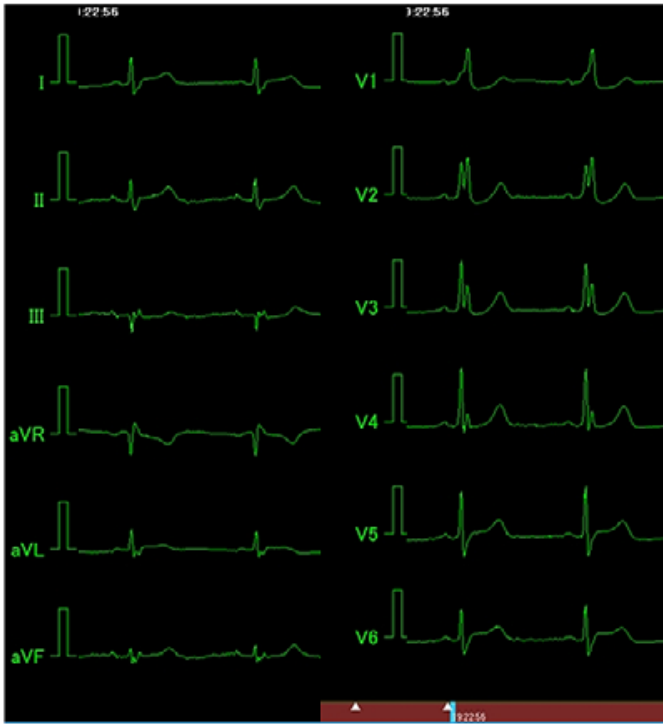

Figure 3. Twelve-lead electrocardiograms before and after pulseless electrical activity (PEA). Note that there are no significant changes in the heart rate or 12-lead electrocardiograms before (left figure) and after (right figure) the PEA. A few minutes after a sudden drop in the blood pressure, a heart rate reduction, and asystole occur.
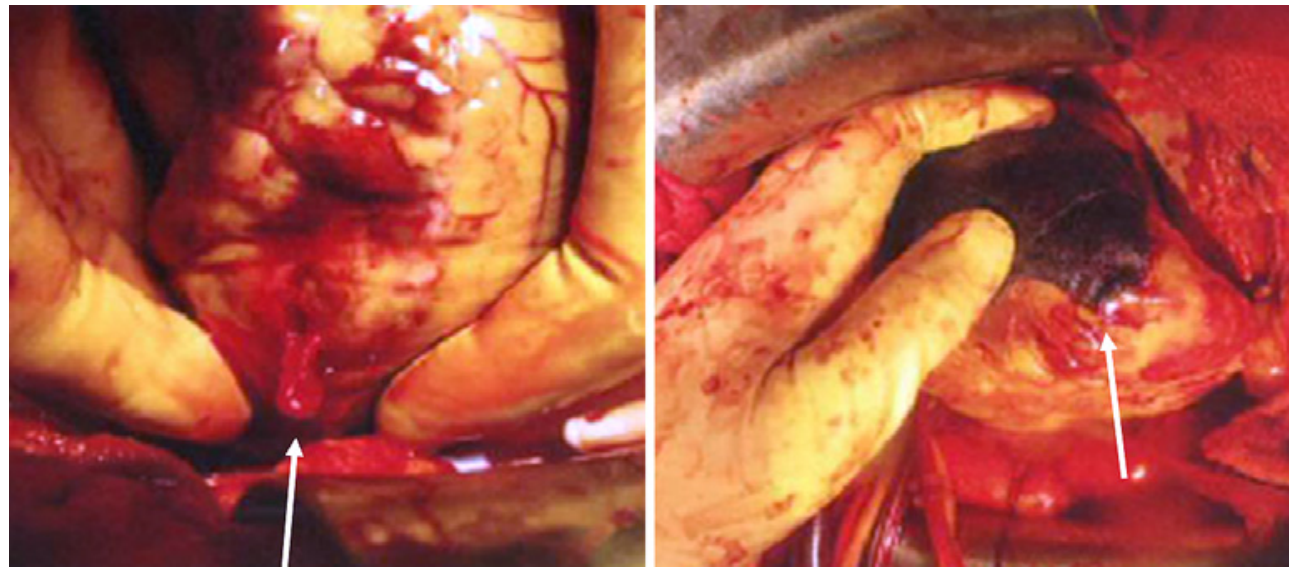

Figure 4. Intraoperative findings of a $2-\mathrm{cm}$ rupture hole blowing blood out into the peripheral myocardium of the left circumflex coronary artery (arrow in the left figure) and a direct closure at the same site (arrow in the right figure).

over, as even case reports are limited in the previous studies about BO-LVFWRs, there has been a lack of consensus on a rapid diagnosis and treatment strategy. We experienced a rare case of a BO-LVFWR successfully treated without any serious cerebral damage despite its occurrence during a PCI for an AMI.

Clinical studies have reported that the frequency of LVFWRs is $1-4 \%$ of patients after an AMI, but it accounts for $10-20 \%$ of patient deaths. ${ }^{3,4}$ LVFWRs occur within the first 5 days after an AMI in about half of the cases and within 2 weeks in over $90 \%$ of patients. ${ }^{5-7)}$ Nakatsuchi $Y$ et al. divided LVFWRs into an early phase $\left(<72\right.$ hours) and late phase (> 96 hours) ${ }^{8)}$ The risk factors for an LVFWR were an older age ( $>65$ years), fe- male gender, hypertension, one-vessel disease (usually a totally occluded vessel with poor collateral circulation), first AMI, absence of reperfusion therapy, and delayed admission. ${ }^{2)}$ LVFWRs occur mainly in the left ventricle with a fairly even distribution between the anterior, inferior, and lateral walls.9) In the present case, it occurred in the early phase, and the risk factors for an LVFWR included hypertension, and one-vessel disease with a total occlusion and poor collateral circulation.

In this case, his life was saved without any serious cerebral damage through a prompt diagnosis by echocardiography and appropriate treatment, including the insertion of mechanical support and pericardiocentesis, leading to maintaining the VA-ECMO flow until the operation. 
Transthoracic echocardiography is the most important diagnostic tool for LVFWRs, with a sensitivity and specificity of the diagnosis between $93 \%$ and $98 \% .^{10}$ The detailed onset pattern of the BO-LVFWR could be observed because it occurred during the PCI. That is, the blood pressure suddenly decreased during the PEA within seconds, i.e. electromechanical dissociation, in spite of having no significant change in the 12-lead ECG or heart rate. The pulse slowed down, and asystole developed a few minutes after the sudden drop in the blood pressure. These findings provided the clinical importance of continuous blood pressure monitoring in AMI patients with the risk of an LVFWR, because it would be too late to respond after the pulse had slowed. It was considered that an immediate confirmation of a pericardial effusion by echocardiography is the most important diagnostic method for a BOLVFWR in this situation. Furthermore, the prompt use of mechanical support (VA-ECMO and IABP) is needed. In such a situation, maintaining the VA-ECMO flow requires the constant release of the cardiac tamponade and securing of the blood vessel volume. In this case, because blood transfusions were not in time, the bloody epicardial fluid from the pericardial drain was administered intravenously to maintain the VA-ECMO flow, and it managed to maintain the circulation until the surgery. Although the pericardial fluid drainage was returned directly to the vein, there were few significant demerits, and it helped maintain the VA-ECMO flow.

An increased PCI rate, better control of the blood pressure, and use of beta-blockers and ACE inhibitors (ACEI)/ARB have contributed to a lower incidence and death rate from LVFWRs. ${ }^{11,12}$ The utilization of betablockers and ACEIs/ARBs in the AMI treatment strategy is correlated with a higher in-hospital survival rate from LVFWRs, especially in LVFWR cases that occur in the late phase. ${ }^{13)}$ In this case, the uncontrolled high blood pressure during the PCI might have contributed to the BO-LVFWR, and the careful introduction of the betablockers and ARBs during the perioperative period was performed, even though this BO-LVFWR occurred in the early phase. Additionally, it was unclear whether those medications could have decreased his mortality as in the late phase.

\section{Conclusion}

Here, we experienced a surviving case from a BOLVFWR occurring during a PCI for an AMI. The present case initially had a sudden blood pressure drop within seconds without any change in the heart rate or 12-lead ECG when the BO-LVFWR occurred. After a few minutes, a heart rate reduction and asystole occurred. These findings suggested that continuous vital sign monitoring could be useful for an early detection of a BO-LVFWR and that an early diagnosis by echocardiography and maintaining the circulation by VA-ECMO until the operation could be lifesaving.

\section{Acknowledgments}

We thank all the doctors and medical staff who were involved in the treatment of this patient.

\section{Disclosure}

Conflicts of interest: Yasuo Okumura received research funding from Boston Scientific Japan.

\section{References}

1. Naeim F, De la Maza LM, Robbins SL. Cardiac rupture during myocardial infarction. A review of 44 cases. Circulation 1972; 45: 1231-9.

2. Yang D, Xiang M, Liu X, He A. Left ventricular free-wall rupture in acute myocardial infarction: a blow-out type case. High Blood Press Cardiovasc Prev 2009; 16: 201-4.

3. Pollak H, Nobis H, Mlczoch J. Frequency of left ventricular free wall rupture complicating acute myocardial infarction since the advent of thrombolysis. Am J Cardiol 1994; 74: 184-6.

4. Rasmussen S, Leth A, Kjøller E, Pedersen A. Cardiac rupture in acute myocardial infarction. A review of 72 consecutive cases. Acta Med Scand 1979; 205: 11-6.

5. Purcaro A, Costantini C, Ciampani N, et al. Diagnostic criteria and management of subacute ventricular free wall rupture complicating acute myocardial infarction. Am J Cardiol 1997; 80: 397-405.

6. Oliva PB, Hammill SC, Edwards WD. Cardiac rupture, a clinically predictable complication of acute myocardial infarction: report of 70 cases with clinicopathologic correlations. J Am Coll Cardiol 1993; 22: 720-6.

7. Lopez-Sendon J, Gonzalez A, Lopez de Sa E, et al. Diagnosis of subacute ventricular wall rupture after acute myocardial infarction: sensitivity and specificity of clinical, hemodynamic and echocardiographic criteria. J Am Coll Cardiol 1992; 19: 1145-53.

8. Nakatsuchi Y, Minamino T, Fujii K, Negoro S. Clinicopathological characterization of cardiac free wall rupture in patients with acute myocardial infarction: difference between early and late phase rupture. Int J Cardiol 1994; 47: S33-8.

9. Cheriex EC, de Swart H, Dijkman LW, et al. Myocardial rupture after myocardial infarction is related to the perfusion status of the infarct-related coronary artery. Am Heart J 1995; 129: 64450 .

10. Pollak H, Diez W, Spiel R, Enenkel W, Mlczoch J. Early diagnosis of subacute free wall rupture complicating acute myocardial infarction. Eur Heart J 1993; 14: 640-8.

11. Figueras J, Alcalde O, Barrabes JA, et al. Changes in hospital mortality rates in 425 patients with acute ST-elevation myocardial infarction and cardiac rupture over a 30-year period. Circulation 2008; 118: 2783-9.

12. Sulzgruber P, El-Hamid F, Koller L, et al. Long-term outcome and risk prediction in patients suffering acute myocardial infarction complicated by post-infarction cardiac rupture. Int J Cardiol 2017; 227: 399-403.

13. Lou B, Luo Y, Hao X, et al. Clinical characteristics and protective factors in patients with acute myocardial infarction undergoing in-hospital myocardial free wall rupture: a single-center, retrospective analysis. J Investig Med 2019; 67: 1097-102. 